\title{
Gerencia cuántica como estrategia para el desarrollo de la espiritualidad a través de los neurotalentos
}

\section{Quantum management as a strategy for the development of spirituality through neurotalents}

\author{
Luis Eduardo Urrego-Cruz \\ lurrego2@unisimonbolivar.edu.co \\ Universidad Simón Bolívar. \\ Colombia \\ https://orcid.org/0000-0002-9210-4338
}

Recibido: 28 de enero de 2020

Revisado: 14 de marzo de 2020

Aprobado: 02 de abril de 2020

Publicado: 19 de mayo de 2020

\begin{abstract}
RESUMEN
El propósito fundamental de esta investigación fue comprender la gerencia cuántica como estrategia para el desarrollo de la espiritualidad a través de los neurotalentos. Teniendo en cuenta teorías de Robles (2014), Bravo (2016), Marques (2010), entre otros, además aportes que hagan los informantes así como la opinión del mismo investigador. Basada desde la perspectiva del método fenomenológico hermenéuticointerpretativo, tomando en cuenta su naturaleza cualitativa de la investigación. La información se obtuvo mediante la observación y entrevistas semiestructuradas a (4) miembros de la Corporación Universitaria Americana Sede Barranquilla. Los resultados indicaron que cuando un gerente debe tomar decisiones tiene en su mente todas las opciones a las que tiene acceso, está el espectro completo pasando por las justificaciones más razonadas hasta las más emocionales, todos estos factores si bien en la realidad se ven aislados, existe un lugar donde están todos comprimidos, ese espacio es la mente, el principio de singularidad.
\end{abstract}

Descriptores: Gerencia cuántica; estrategia; espiritualidad; desarrollo; neurotalentos.

\begin{abstract}
The fundamental purpose of this research was to understand quantum management as a strategy for the development of spirituality through neurotalents. Taking into account theories of Robles (2014), Bravo (2016), Marques (2010), among others, as well as contributions made by the informants as well as the opinion of the same
\end{abstract}




\section{CIENCIAMATRIA}

Revista Interdisciplinaria de Humanidades, Educación, Ciencia y Tecnología

Año VI. Vol. VI. N¹1. Julio - Diciembre 2020

Hecho el depósito de ley: pp201602FA4721

ISSN-L: 2542-3029; ISSN: 2610-802X

Universidad Nacional Experimental Francisco de Miranda (UNEFM). Santa Ana de Coro. Venezuela

\section{Luis Eduardo Urrego-Cruz}

researcher. Based on the perspective of the hermeneutic-interpretive phenomenological method, taking into account its qualitative nature of research. The information was obtained through observation and semi-structured interviews with (4) members of the American University Corporation Sede Barranquilla. The results indicated that when a manager has to make decisions he has in mind all the options to which he has access, there is the whole spectrum going through the most reasoned justifications to the most emotional, all these factors although in reality they are isolated, there is a place where they are all compressed, that space is the mind, the principle of singularity.

Descriptors: Quantum management; strategy; spirituality; developing; neurotalent.

\section{INTRODUCCIÓN}

Es innegable la pérdida tanto del sentido humano como lo ambiental en la sociedad, gran parte del problema lo ocasionan las organizaciones donde las políticas, misión, visión, no se orientan al desarrollo humano sostenible y sustentable, sino que cada vez enfocan más sus esfuerzos hacia la generación de dinero, por lo tanto, es mayor la fisura entre capital- hombre - bienestar.

Desde esta perspectiva, ante el hecho que cada día se desarrollan más organizaciones modernas, surge, a partir del año 1900 con la introducción por parte del físico alemán Max Planck (1858-1947) la teoría cuántica, un marco conceptual innovador que permitirá el desarrollo de la organización post-moderna, la cual requerirá, entonces, estar conformada por quienes asuman un pensamiento probabilístico, dejando atrás lo determinístico, buscando en las instituciones el comportamiento como seres vivos más no como máquinas.

Dentro del mismo contexto, la gerencia cuántica, necesita un gerente comprensivo, activando energías en sus trabajadores al momento de intervenir y sea sustituido por la que inicialmente tenían, para lograr la actividad, por tanto, los estudios gerenciales del comportamiento de grupos, tienden a manifestar una conducta diferente cuando los individuos son observados, esto da a lugar a la ética empresarial, dando lugar a una del patrono y otra del trabajador.

Lo anterior se explica, cuando un individuo no puede considerar su trabajo totalmente satisfecho porque cuando se evalúa a sí mismo, intervienen factores emocionales, 
impidiendo una verdadera valoración objetiva sobre su participación, así como la intervención de creencias y emociones, haciendo de la realidad no la que se plasma en un papel sino la perspectiva de mente a quienes participan.

Por tanto, cuando se habla sobre gerencia cuántica se debe decidir evaluando desde la mente todo el abanico de opciones, desde el espectro más completo pasando por justificaciones razonadas hasta las más emocionales, así como todos los factores aislados, sin embargo, existe un lugar donde están comprimidos y ese espacio no es otro que la mente de los gerentes.

Es así, la gerencia cuántica, no abandona la actitud para preparar profesionales, sobre todo en las áreas gerenciales ha olvidado la actitud como investigador que estos deben tener, se preparan profesionales para ocupar cargos o para mantenerse en estos, adquirir cierta actualización, buscar oportunidades de mejoras salariales, entre otros.

De acuerdo a lo descrito, la investigación persigue comprender la gerencia cuántica como estrategia para el desarrollo de la espiritualidad a través de los neurotalentos, la explicación sobre los elementos concernientes a este enfoque pueden estar presentes en todo el estudio, requiriendo argumentarse desde un referente conceptual, además que profundiza en la percepción sobre los miembros en las organizaciones a estudiar.

\section{Desarrollo Teórico}

\section{Modelo de Gerencia Cuántica}

Todo gerente debe entender que al intervenir en sus empleados aumenta la energía de los mismos, diferente a la que poseían durante el inicio de sus actividades, esto lo indica los estudios gerenciales del comportamiento de grupos o personas, por lo general estos tienden a manifestar conductas cuando son observados por su jefe haciendo ver que trabajan mucho.

De allí entonces, es difícil medir con precisión el nivel de eficiencia en los trabajadores, cuando no se observan trabajando bajos sus criterios, esto sería la autonomía del trabajador, cuando está siendo observado o evaluado trabaja bajo los criterios organizacionales, políticas y procedimientos organizacionales, ésta da a lugar a la 


\section{Luis Eduardo Urrego-Cruz}

ética empresarial, donde hay una ética tanto del patrono como del empleado, el conocido principio de observación.

Por ello, no puede considerar su trabajo totalmente satisfecho, cuando se evalúa a sí mismo, intervienen factores emocionales, impidiendo una verdadera valoración objetiva en su participación, así como creencias, emociones de cada grupo interviniendo así la realidad no sólo la plasmada en papel sino desde la perspectiva de los que participan, entendiéndose esto como el principio de incertidumbre.

En este sentido, cuando un gerente debe tomar decisiones tiene en su mente todas las opciones a las que tiene acceso, está el espectro completo pasa por las justificaciones más razonadas hasta las más emocionales, todos estos factores si bien en la realidad se ven aislados, existe un lugar donde están todos comprimidos, ese espacio es la mente de los gerentes, o el principio de singularidad.

Cuando se decide emerge una realidad, explica Schrödinger (2015), gerencialmente, están las posibilidades, cuando se decantan por una opción, todas las demás opciones se desaparecen, emergiendo así una la realidad, cuando se habla de decisiones en inversiones por ejemplo, se dice que un proyecto tendrá una rentabilidad "a" y otro proyecto tendrá una rentabilidad "b", ambos proyectos son ejecutables, "a" puede ser mayor a "b", económicamente a lo mejor sea mejor a pero quizás mejor es más cómodo b, así los dos proyectos son posibles, pero solo una será realidad cuando se decida por uno o por otro.

Por lo anterior, un gerente que posea estos conocimientos científicos no puede abandonar esa actitud, la preparación de profesionales en los postgrados, sobre todo en las áreas gerenciales ha olvidado un poco el rol como investigador, preparan profesionales para ocupar cargos o para mantenerse en estos, adquirir cierta actualización, buscar oportunidades en mejoras salariales, otros.

Explica Méndez (2006), se ha olvidado la acción como científicos sociales pueden desempeñar, además las empresas son una fuente importante, por cuanto suministran la información necesaria para construir modelos que puedan aplicarse a otras organizaciones, por ello, la investigación colaborará con la organización en dos niveles el primero referido a buscar la eficacia y eficiencia en los procesos de la organización, 
segundo referido a la documentación requerida para cultivar la enseñanza o la formación de profesionales en el área gerencial.

\section{Elementos de la Gerencia Cuántica}

Desde la perspectiva de Bravo (2016), la gerencia cuántica posee tres principios indispensables, elementos claves para el desarrollo potencial del gerente en sus funciones, desarrollando una ciencia para cada elemento en el trabajo, el cual reemplaza el viejo método, así científicamente seleccionan, entrenan, enseñan, desarrollan a los trabajadores, sin importar como se haya hecho en el pasado y que tan buenos hayan sido entrenados; cooperan con el fin de asegurar el trabajo centrado en los principios científicos desarrollados.

El primer principio Incertidumbre, a nivel organizacional, es un concepto crucial para la comprensión de los fenómenos económicos. Esta supone, por ejemplo, los equilibrios en un mercado cada vez más competitivo, se alcanzan cuando cada uno los agentes económicos en la empresa posean la información completa sobre los intercambios producidos en el mismo. En otros términos, se parte del supuesto de que tanto productores como consumidores poseen el conocimiento necesario para maximizar sus beneficios.

Sin embargo, en el mundo real, los empresarios desconocen la cantidad, costo, precio exacto para poder vender bienes y los consumidores no tienen una idea precisa de la utilidad proporcionada por ellos que adquieran, para poder deben trabajar con valores aproximados. Además, la falta de información necesaria no obedece sólo a problemas derivados en la complejidad económica, sino más bien prever la reacción en otros agentes intervinientes del mercado.

Esto, aunado a la incertidumbre de las variables del mundo físico, haciendo ella misma un problema irreductible, pues no es posible conocer todos los riesgos asociados con una decisión cualquiera. Su existencia produce el desconocimiento de resultados o acciones, puede suceder algo diferente a lo esperado. Los individuos deben escoger constantemente entre diversos cursos de acción resultados posibles. El problema 


\section{CIENCIAMATRIA}

Revista Interdisciplinaria de Humanidades, Educación, Ciencia y Tecnología

Año VI. Vol. VI. N¹1. Julio - Diciembre 2020

Hecho el depósito de ley: pp201602FA4721

ISSN-L: 2542-3029; ISSN: 2610-802X

Universidad Nacional Experimental Francisco de Miranda (UNEFM). Santa Ana de Coro. Venezuela

Luis Eduardo Urrego-Cruz

consiste en determinar el modo en que, en tales condiciones, los individuos toman sus decisiones.

La solución tradicional al problema se basaba en la teoría de las probabilidades, ésta consiste en demostrar si el evento tratado ha ocurrido en varias oportunidades para así demostrar los resultados posibles. Sin embargo, la teoría no tiene mayor aplicación si no pueden repetirse (o no se han repetido hasta el momento) las acciones a estudiar; y esto es, precisamente, lo que ocurre con una gran cantidad de decisiones económicas a tomar en el mundo real.

Como segundo principio está la complementariedad, este principio tiene una gran incidencia, en la comprensión racional el cual es imposible entender en su totalidad la realidad de la naturaleza, con ello se está marcando un límite al pensamiento en su relación con el mundo físico, una limitación con carácter externo, un límite físico interpuesto por el hecho que el conocimiento humano se rige también, como sus objetos, por leyes naturales.

En este sentido, se puede asegurar por este principio que, en los asuntos humanos es imposible acotar la realidad a una sola perspectiva que la capture en forma integral. A nivel de estrategias, la decisión más rica en el seno organizacional, integra en un todo coherente los aportes individuales, tomando en consideración los aportes más disimiles, complementando así las distintas visiones estratégicas.

El principio de complementariedad señala la realidad desbordada a las explicaciones, el juego intelectual consiste en la imposibilidad de conocer la totalidad. La realidad en la organización empresarial es transmitida por distintos medios, los cuales permiten ver la posición entre otros, generando complementariedad, riqueza, en sinergia con otras opiniones.

Asimismo, cada persona posee un background único, el cual le ayuda a tomar posiciones para comprender el mundo y, su permanente diálogo con personas con opiniones distintas, aumenta el conocimiento complejo. El tercer principio son la Fluctuaciones y Bifurcaciones, desde la perspectiva del desorden como generador de orden, estos son factores importantes para las organizaciones empresariales postmodernas. Estos elementos cuánticos adquieren importancia, por cuanto los 
fenómenos tanto naturales como sociales en la vida real son infaliblemente no lineales. En primera instancia, las fluctuaciones provienen de la misma aleatoriedad del sistema, son aquellos movimientos generadores de variaciones (cambios), producidos cerca de un punto de bifurcación y pueden hacer que un sistema como las organizaciones empresariales tome rumbos diferentes al tomado de no hacerlo así. En una organización, al enfrentar una situación de crisis se puede presentar un punto bifurcantes ante el cual debe haber más esfuerzo, más energía, para trazar nuevas estrategias implicando un crecimiento en la estructura funcional de la organización. Éste constituye un umbral estable en la estructura organizacional puede derrumbarse, o bien trascender hacia uno o varios nuevos estados.

De manera positiva, pensar en el no equilibrio permite abrir las posibilidades organizacionales. La organización en el no equilibrio es un sistema sensible, inestable, caótico, y dentro de la misma dinámica elimina escenarios posibles que representan cambios, es decir, un nuevo orden con mayor complejidad. Por lo tanto, la organización como estructura está abierta al entorno para adquirir una nueva sensibilidad empresarial.

\section{La Espiritualidad}

En lo últimos años se ha tratado de establecer la espiritualidad en relación a la productividad y el liderazgo empresarial como estrategias gerenciales. Robles (2014), expone cómo los empresarios o altos ejecutivos definen el rol de espiritualidad como una estrategia gerencial aplicada a la cotidianidad, además de los factores significantes o propósitos.

Por otro lado, Bolman y Deal (2014, p. 190), dan una visión de liderazgo al afirmar "el corazón de un verdadero líder solo puede ser encontrado en el corazón de un líder", redefiniendo la espiritualidad como una continua búsqueda en la optimización sobre la productividad en una organización sin olvidar las ciencias sociales aplicadas a la espiritualidad en cualquier actividad laboral y cotidiana.

Desde esta perspectiva, la preocupación por la espiritualidad en estrategias o temas gerenciales nace únicamente por la ocupación con la deshumanización y la 


\section{Luis Eduardo Urrego-Cruz}

instrumentalización de los seres humanos en la sociedad, conllevando a múltiples paradojas relacionadas con que si estos estudios sobre espiritualidad empresarial pueden o no trascender en la historia $u$ aplicabilidad empresarial.

Para afianzar este estudio Robles (2014), define espiritualidad como un sentimiento fundamental de estar entrelazado e interconectado con su ser completo, con otros o con el universo entero, es vista no como la autorrealización del personal laborando sino como una estrategia de negocios que no desliga la deshumanización con el capitalismo agresivo.

Recientemente Espinosa (2015), expreso la espiritualidad es el deseo en descubrir nuestro propósito final en la vida, el verdadero sentido final en la vida o el verdadero sentido que tiene a vivir de acuerdo con ella, implica que, la espiritualidad es el significado de la vida o propósito final, enmarcado en un contexto de cómo se lleva.

\section{Rol del Gerente Cuántico desde la Espiritualidad}

La importancia de la espiritualidad en la gerencia, es inminente sobre todo cuando el líder es quien direcciona los procesos organizativos en la organización, ya que, los emprendedores deben ejercitar lo expresado como fe y hacerlo casi a diario, lo cual no sucede solamente como consecuencia a los incentivos en ventas, indicadores sobre productividad, ahorro, o lo elegido para premiar; sucede también por lo transmitido por los líderes, enfrentarse tanto a lo bueno o malo, frente a lo próspero o adverso, a todos los aspectos que hacen a una organización desde el punto de vista de las relaciones humanas.

Este es un punto de vista muy claro señala Oromí, (2015), al plantear una organización está formada por personas, por seres humanos que aportan conocimientos a una organización, donde todos colaboran por un objetivo en común, cuyos valores personales prevalecen sobre los procesos organizativos, convirtiéndolos en valores organizaciones.

Entonces ese objetivo en la vida de las personas se pregunta todos los días, ¿hacia dónde se va? ¿Qué se quiere lograr? ¿Hasta dónde se quiere llegar?, son preguntas quizás sencillas, pero con un gran contenido en autoconocimiento que claramente no 


\section{Luis Eduardo Urrego-Cruz}

se pueden responder si no se hace un análisis claro sobre valores, principios, fortalezas y debilidades propias.

Por lo anterior, Dolan (2015, p. 8), plantea no puede existir una verdadero liderazgo espiritual si se carece de valores morales, él lo describe como un "Liderazgo Visionario", describiéndolo como "una perspectiva que a menudo se relaciona con la capacidad o la propensión a ver fuerzas superiores espirituales en acción detrás de los sucesos normales", es decir, los líderes son quienes deben unir la espiritualidad con los valores, el liderazgo nato, alineándolos con la capacidad de pensar globalmente o la ambición para lograr las metas, no deben sobrepasar los valores humanos y la moral propia al decidir éticamente lo mejor para sí mismo o para la organización.

Pero para la sociedad en general el valor predominante una organización es la honestidad, definida como un valor o cualidad propio de los seres humanos, tiene una estrecha relación con los principios de verdad y justicia y con la integridad moral. Una persona honesta es aquella que procura siempre anteponer la verdad en sus pensamientos, expresiones o acciones.

Desde la visión de Drake (2008), hace una fuerte crítica a los valores morales y éticos organizacionales del mundo al decir a las empresas diciendo "tengan confianza en nosotros", las personas están pidiendo muestren las cuentas como pruebas de la honestidad e integridad. Para este autor la postura de espiritualidad y liderazgo va unida, pero ese lazo entre los dos se llama honestidad o ética de negocios, reconociendo no es la organización quien establece los valores y la moral, sino los individuos dentro de la organización que tiene valores.

Para Dolan (2015), la postura de la espiritualidad en el trabajo es muy clara, un lugar de trabajo espiritualmente amigable respeta las creencias más profundas en la gente incrédula, permitiendo alentar a usar esas creencias como recurso a incorporar estos valores en lo que hacen y en como realizan su trabajo, indicando no son solo las creencias religiosas si no los valores que cada ser humano constituye como principio de vida. 


\section{METODOLOGÍA}

Ante la postura adquirida, la Epistemología acarrea una constante redefinición de sus paradigmas; particularmente el investigador es consciente de la confrontación entre lo objetivo y lo subjetivo, siendo consecuente en el criterio de que no hay una verdad absoluta, por lo tanto, al describir el contexto de manera acertada se requiere de una visión holística, Martínez (2010), refiere es necesario generar cambios en la manera de pensar, percibir y valorar el avance del conocimiento.

Desde la perspectiva de Morín (2012) un paradigma científico, se conoce como un principio de distinciones, relaciones, oposiciones fundamentales entre las nociones centrales, las cuales generan y controlan el pensamiento. Este paradigma, permite percepciones que están influencias por la globalización, tecnología y valores, que obligan a renovar o innovar en nuevos paradigmas. Se menciona, además el paradigma cualitativo definido por Blaxter, Hughes y Tight (2008) los cuales indican que se da como una forma de clasificar las creencias y visiones más complejas del mundo, en otras palabras, como la elección del camino para investigar correctamente. De lo anterior se desprende, que la investigación de corte cualitativo, recurre al paradigma postpositivista, en el cual según Sautu (2005) solo se puede conocer la realidad de manera probabilística e imperfecta, manteniéndose la objetividad como algo ideal, no obstante al imaginar que todos los métodos poseen las mismas características; por lo que Denzin \& Lincoln, (2005) acompañan la idea de que este tipo de investigación cualitativa, puede resolver situaciones problemáticas, debido a que contemplan las interpretaciones del mundo, dentro de sus muchas variantes se encuentra la Teoría Fundamentada.

Bajo las consideraciones anteriores, se hace uso del interaccionismo simbólico dentro del proceso hermenéutico o interpretativo, el cual de acuerdo con Blumer (2009) en un proceso en el cual los humanos interactúan con símbolos para construir significados, donde la mente humana, define el análisis de la acción humana, en correspondencia Heidegger (2014, p.126) señala que es "una ciencia interpretativa en busca de leyes" es por ello que intenta representar, así como comprender el proceso de creación y asignación de significado al mundo real. 
Asimismo, la perspectiva hermenéutica, por su naturaleza cualitativa, necesita de un diseño general adecuado al arte para interpretar, a medida que vaya identificando la realidad objeto $u$ estudio en la interacción con los sujetos que cohabitan en el contexto, guie el estudio a la consecución de sus objetivos para dar respuesta a la problemática planteada; en ese sentido, Bonilla- Castro y Rodríguez (2005, p.127), manifiestan "los diseños cualitativos son interactivos, dinámicos, emergentes; en ellos, la exploración del problema, la recolección de los datos, el análisis, la interpretación, la validación están inextricablemente tejidos a lo largo de la investigación".

Con base en esta cosmovisión epistémica asumida, la presente investigación se orientará desde el método fenomenológico hermenéutico; por lo tanto, mi preocupación se centra en establecer cómo los docentes conocen, interpretan y comprenden el fenómeno considerando las construcciones sociales que sobre ello han elaborado desde las subjetividades e intersubjetividades en el mundo vivido, desde ese estar ahí, como advierte Heidegger (2014), las estructuras sociales y culturales. Luego, estimo lo congruente con la realidad socio-contextual de las actividades vivenciales dentro de la Institución Corporación Universitaria Americana.

Es por ello que para Martínez (2010), el estudio no puede consistir solamente en describir y analizar, sino también, y más importante aún, sintetizar, organizar, componer, estructurar la información, reconociendo la importancia de develar el significado para su interpretación, ir más allá de los actos físicos, considerando de este modo la importancia que tienen las definiciones intencionales, motivacionales que enfatizan en el significado y las relaciones entre estos y no los meros aspectos materiales de la estructura, que de otros modos inconexos e incomprensibles.

En la presente investigación, la compresión de la realidad como de sus múltiples dimensiones, dinamismos y relaciones, convierten el foco de estudio en un fenómeno de naturaleza compleja, comprendido por quienes conviven en el contexto de estudio, desde sus propias vidas y perspectivas, de cuyo análisis emergerán las categorías para ser interpretadas, a la luz de una dialógica de significados compartidos

Destaco que el término fenomenología ha sido utilizado como paradigma, filosofía, perspectiva y método cualitativo de investigación. Sobre ello, Valles (2009) considera 


\section{Luis Eduardo Urrego-Cruz}

insostenible considerar la fenomenología como perspectiva o una perspectiva más. El autor lo justifica en condición de filosofía, disciplina de donde surge la aproximación fenomenológica como corriente de conocimiento, de conocer y comprender la realidad. De igual modo, justifica su planteamiento en que la fenomenología es y ha sido raíz intelectual de muchas otras perspectivas y estilos cualitativos de investigación.

La fenomenología es una corriente de pensamiento que surge con el propósito de estudiar las realidades humanas, tal como ellas son, dejando que se manifiesten por sí mismas, sin forzar, alterar o constreñir su naturalidad y dinamicidad. Por el contrario, la fenomenología pide al investigador reflexionar, respetándola, valorándola en su totalidad como en sus aspectos constituyentes y configurativos. la fenomenología, cuyo origen es de orden filosófico según Valles (2009), es la base epistémica de la forma de conocer la realidad desde otras diversas aproximaciones cualitativas, parte de la realidad tal como ella es y se presenta, para desde el conjunto, describirla, interpretarla y comprenderla.

Otro aspecto fundamental a tener en cuenta, es que no se tratará de un análisis mecánico en los datos o procedimientos sobre la interpretación de la información, sino se verá orgánica así como estratégicamente la realidad; adquiriendo una forma de pensar sobre los datos, entendiendo hacia dónde se quiere ir; recorriendo un trayecto hermenéutico desde los datos a lo abstracto, de lo abstracto a los datos nuevamente, dándose un ciclo interpretativo, el cual analíticamente genere una nueva teoría.

En este sentido, ese camino necesitará en primer lugar, hacer trabajo mental desde un pensamiento concreto a un pensamiento abstracto; es decir, desplegar la capacidad para observar, no solamente lo que se muestra ante los sentidos, sino ver los datos de manera ordinaria y abrir la mente a la posibilidad en ver más allá de los datos obtenidos. Así pues, el método aplicado en esta investigación, se basará en la sistematización lógica de las interpretaciones del significado que broten sobre las interacciones con los sujetos en una realidad ya seleccionada para tal fin; la institución seleccionada y su contexto.

Es decir, el trabajo consistirá en interpretar las teorías las cuales puedan originar la 


\section{Luis Eduardo Urrego-Cruz}

teoría sustantiva sobre la Gerencia Cuántica como estrategia para el desarrollo de la espiritualidad a través del neurotalento, para descubrir sus significados, comprendiendo lo mejor posible comportamientos, escritos, palabras, valores, otros. Las cuales se observarán en el escenario, para llegar a la argumentación científica de la teoría emergente, desde una lógica interactiva, conservando la singularidad del contexto del cual forma parte.

\section{HALLAZGOS DE LA INVESTIGACIÓN}

Como propósito principal el investigador planeó comprender la gerencia cuántica como estrategia desde la espiritualidad para el desarrollo de los neurotalentos, cuya población en estudio estuvo conformada por personas involucradas al contexto gerencial, finalmente se dio respuesta a las interrogantes del estudio para poder dar respuesta a los propósitos planteados al inicio y así poder generar una teoría sustantiva que contribuirá a solventar el fenómeno en estudio.

Para ello se plantean los hallazgos originados:

Primer Hallazgo: La Gerencia cuántica es la predicción del comportamiento futuro en un sistema dinámico en términos de una función por onda prediciendo analíticamente la probabilidad precisa sobre los eventos o resultados.

Segundo Hallazgo: Los elementos básicos de la gerencia cuántica son producto, precio, plaza, promoción y estos mezclados con otros elementos garantizan unos resultados óptimos.

Tercer Hallazgo: En cuanto a la gerencia cuántica hay que mirar la fusión e intercesión entre el pensamiento crítico y física cuántica para poder producirse un cambio trascendental.

Cuarto Hallazgo: El hombre siempre ha buscado nuevos caminos, ésta es una estrategia donde prevalece la intuición, es importante también mirar la creatividad para ser visionario, de allí entonces como la gerencia cuántica busca el desarrollo de las habilidades del pensamiento, desde la participación global, dónde, holísticamente todos aporten y en donde se sepa identificar ese pensamiento, que no todos tenemos. Quinto Hallazgo: La espiritualidad es una intención de las personas, no debe 


\section{CIENCIAMATRIA}

Revista Interdisciplinaria de Humanidades, Educación, Ciencia y Tecnología

Año VI. Vol. VI. N¹1. Julio - Diciembre 2020

Hecho el depósito de ley: pp201602FA4721

ISSN-L: 2542-3029; ISSN: 2610-802X

Universidad Nacional Experimental Francisco de Miranda (UNEFM). Santa Ana de Coro. Venezuela

Luis Eduardo Urrego-Cruz

confundirse con cristianidad. Es necesario mirar cual es la visión de la empresa centrada en la parte humanística de la formación del ser humano.

Sexto Hallazgo: Los neurotalentos son obviamente, los pensamientos de las siete inteligencias múltiples, en estos momentos se divide en cómo está conformado nuestro cerebro; nunca va a permitir tener temores ni riesgos sino simplemente avanzan para realizar las metas y los logros.

Séptimo Hallazgo: Los elementos de la gerencia cuántica se basan en los procesos administrativos planear, organizar, dirigir y controlar que sumados a otros como son producto, precio, plaza promoción pueden generar beneficios a las organizaciones.

Octavo Hallazgo: Para que exista espiritualidad deben existir lideres convencidos de lo que hacen y quieren hacer para evitar caer en contradicciones.

Noveno Hallazgo: Existe divisiones en el cerebro que las personas no las desarrollan y son las necesarias para poder tomar decisiones.

Décimo Hallazgo: El rol del gerente cuántico es identificar los talentos, combinar estos talentos, pasiones, conocimiento, destreza; para poder crear fortalezas ayudando a encontrar oportunidades. Cuando todo esto se conecta, se va a logrando una excelencia profesional y un éxito empresarial.

Decimoprimer Hallazgo: La gerencia cuántica es el estudio de las conexiones entre personas aplicadas a la organización para obtener tanto indicadores de gestión como el cumplimiento en los objetivos.

Decimosegundo Hallazgo: El efecto de la espiritual en la producción es grande, no existe proyecto sin motivación, sin entusiasmo, entonces el éxito del proyecto estaría profundamente vinculada al compromiso que tengan los integrantes en el proyecto.

Decimotercer Hallazgo: Para determinar la dominancia cerebral deben estar presentes unas cualidades del cerebro derecho, también debe tener una capacidad analítica importante en su cerebro izquierdo, por ello el gerente debe ser integral y tener contacto con todos los miembros de la organización.

Decimocuarto Hallazgo: El gerente debe tener una intuición pensante en la parte social, desde ahí obviamente lo entrelaza con la espiritualidad, formando seres integrales para consolidar el éxito organizacional. 


\section{CIENCIAMATRIA}

Revista Interdisciplinaria de Humanidades, Educación, Ciencia y Tecnología

Año VI. Vol. VI. N¹1. Julio - Diciembre 2020

Hecho el depósito de ley: pp201602FA4721

ISSN-L: 2542-3029; ISSN: 2610-802X

Universidad Nacional Experimental Francisco de Miranda (UNEFM). Santa Ana de Coro. Venezuela

Luis Eduardo Urrego-Cruz

Decimoquinto Hallazgo: La gerencia cuántica posee tres principios indispensables, para el desarrollo potencial del gerente: entrenar, enseñar y desarrollar a los trabajadores.

Decimosexto Hallazgo: La incertidumbre es un concepto crucial para la comprensión de los fenómenos económicos.

Decimoséptimo Hallazgo: La complementariedad, en el plano organizacional tiene una gran incidencia, por cuanto la comprensión racional le es imposible entender en su totalidad la realidad de la naturaleza.

Decimoctavo Hallazgo: Las fluctuaciones y bifurcaciones, desde la perspectiva del desorden como generador de orden, son factores importantes para las organizaciones empresariales.

\section{REFLEXIONES FINALES}

Al analizar las diferentes posturas cognitivas surgidas en la investigación, se puede reflexionar finalmente que, cuando un gerente debe tomar decisiones tiene en su mente todas las opciones a las que tiene acceso, está el espectro completo pasando por las justificaciones más razonadas hasta las más emocionales, todos estos factores si bien en la realidad se ven aislados, existe un lugar donde están todos comprimidos, ese espacio es la mente de los gerentes, este es el principio de singularidad.

Un gerente poseedor de estos conocimientos científicos no puede abandonar esa actitud, ya que la preparación de profesionales en los postgrados, sobre todo en las áreas gerenciales ha olvidado un poco el rol de investigador, preparan profesionales para ocupar cargos o para mantenerse en estos, adquirir cierta actualización, buscar oportunidades de mejoras salariales, entre otros.

En cuanto a la gerencia cuántica como estrategia desde la espiritualidad el estudio arrojó que existe una relación directa con la productividad y el liderazgo empresarial como logros gerenciales.

Los empresarios o altos ejecutivos definen el rol de espiritualidad como una habilidad gerencial aplicada a la cotidianidad, unida a factores significantes y propósito a la misma. 
La preocupación de la espiritualidad en estrategias o temas gerenciales nace únicamente por la ocupación con la deshumanización y la instrumentalización de los seres humanos en la sociedad, conllevando a pensar si tiene transcendencia 0 aplicabilidad en el área empresarial.

La espiritualidad como sentimiento fundamental debe estar entrelazado e interconectado con su ser completo, con otros y con el universo entero, debe verse como una estrategia de negocios que no desliga la deshumanización con el capitalismo agresivo.

La importancia de la espiritualidad en la gerencia, es inminente cuando el líder es quien direcciona los procesos en la organización, los emprendedores deben ejercitar lo que se expresa con fe y hacerlo casi a diario.

La postura de la espiritualidad en el trabajo es muy clara, un lugar de trabajo espiritualmente amigable respeta las creencias más profundas de la gente, permitiendo alentar a usar esas creencias como recurso a incorporar estos valores, indicando que no son solo las creencias religiosas si no los valores intrinsicos del ser humano.

Una de las estrategias más efectivas, es la espiritualidad, por cuanto aumenta la productividad, la cual va asociada directamente del liderazgo cuando se habla de organizaciones eficientes, la confianza depositada por las personas en un liderazgo solido por parte de sus dirigentes, en definitiva es un indicador confiable de la satisfacción de los empleados, los servidores, los asociados y todos aquellos que participan en la consolidación de los objetivos en una organización.

El gerente cuántico debe considerar desde la espiritualidad, el neurotalento de su personal a cargo, entendiendo que las divisiones cerebrales actúan de manera inconsciente, la persona no se percata, lo único identificado es su personalidad alegando ser siempre de esa manera.

Conocer la dominancia cerebral de una persona permite explicar y aprender a aceptar diferencias individuales, siendo muy buena herramienta para mejorar la efectividad personal y organizacional. 
Por tanto, una organización, con un equipo formado por personas con diferentes modos de pensamiento será sumamente efectivo, pues logrará resultados en forma rápida, sin desgastarse sus miembros, utilizando estrategias como el coaching, y el diagnostico de dominancia cerebral.

\section{REFERENCIAS CONSULTADAS}

Blaxter, L; Hughes, C y Tight, M (2008). Cómo se Investiga. Barcelona: Editorial Grao.

Blumer, H. (2009) El Interaccionismo Simbólico. Barcelona: Hora S. A.

Bolman, Lee. Q; Deal, Terrence. E. (2014). Organización y liderazgo. Wilgminton: Addison-Wesley.

Bravo, V. (2016). ¿Postcoloniales, nosotros? Límites y posibilidades de los estudios postcoloniales. En V. Bravo, El señor de los tristes y otros ensayos (pp.119-138). Caracas, Monte Ávila Editores.

Denzin, N. y Lincoln, Y. (2005). The Sage Handbook of Qualitative Research. Third Edition. London, UK: Sage.

Dolan, L. S. (2015). Coaching por valores. Primera edición. Bogotá, D.C. Colombia. Ediciones de la U.

Drake, B.H. (2008): "Ethical and legal aspects of managing corporate cultures". California Management Review, Vol. 30, N. 2, pp. 107-123.

Espinosa, J (2015) "La espiritualidad ignaciana y su relación con la cristología de karl rahner Algunas consideraciones", en Teología Espiritual LXI. 253-266. Disponible En: https://n9.cl//irgp

Heidegger, M (2014) Los conceptos fundamentales de la metafísica. Mundo, finitud, Soledad. Alianza. Madrid.

Martínez, A. (2010). Los Conceptos de Conocimiento, Epistemología y Paradigma, como Base Diferencial en la Orientación Metodológica del Trabajo de Grado.

Morin, E. (2012). Epistemología de la complejidad. Gazeta de Antropología. 20, artículo 02 - [Artículo en línea], Disponible: http://hdl.handle.net/10481/725 [Consulta: 2019, 2].

Oromí, J (2015) El Liderazgo y los Jefes. En posi+ivo boletín interno de emedical. Diponible en: https://n9.cl/xm92 
Robles, E (2014) Implicaciones de la espiritualidad en la administración de empresas. Revista Empresarial Inter Metro / Inter Metro Business Journal Spring / Vol. 7 No. 1 / p. 29.

Rodríguez, G (2005) Metodología de la Investigación Cualitativa. Málaga, España. Ediciones Aljibe.

Sautu, R. (2005) La integración de métodos cualitativos y cuantitativos para el estudio de las experiencias de corrupción. En: Cinta de Moebio № 13. Facultad de Ciencias Sociales. Universidad de Chile. http://www.moebio.uchile.cl

Schrödinger, E. (2015), ¿Qué es la vida?. Trad. y notas de Ricardo Guerrero. Tusquets, $7^{\mathrm{a}}$ ed. Barcelona. Colección Metatemas.

Valles, M. S. (2009), Técnicas cualitativas de investigación social. Reflexión metodológica y práctica profesional, Madrid, Síntesis. 